\title{
Report on the fourth TDR/IDRI meeting on second generation vaccine against Leishmaniasis. Merida, Yucatan, Mexico, May 1- 3, 2001.
}

Eric Dumonteil ${ }^{1}$, Diane McMahon-Pratt ${ }^{2}$, Virginia L. Price ${ }^{3}$.

${ }^{1}$ Universidad Autónoma de Yucatán, Mérida, Yucatán, Mexico, ${ }^{2}$ Yale University School of Medicine, New Haven, CT, USA, ${ }^{3}$ UNDP/ World Bank/WHO Special Programme in Training and Research in Tropical Diseases (TDR) Geneva, Switzerland.

A meeting entitled "Second generation vaccines against leishmaniasis" was held in Merida, Mexico on May 1-3, 2001. The meeting was hosted by the Universidad Autónoma de Yucatán in Merida and sponsored by the WHO/World Bank/ UNDP Special Programme for Training and Research in Tropical Disease (TDR) and the Infectious Disease Research Institute (IDRI). The meeting consisted of two parts: 1) a review of the evaluation of recombinant Leishmania antigen testing in two independent labs and 2) individual presentations regarding second generation Leishmania vaccine candidates. [The first generation Leishmania vaccine is considered to be the killed whole Leishmania parasite vaccine that has been tested in clinical trials in Iran, Sudan, and Latin America (1-3). A new formulation of this vaccine including bacillus Calmette-Guérin(BCG) and alum as adjuvants will be tested in 2001 in Iran and Sudan]

In an effort to organize a systematic way of testing candidate antigens as potential vaccine candidates, comparative studies were done in two different laboratories; Universidade Federal de Bahia, Salvador, Brazil, and the University of Copenhagen, Copenhagen, Denmark. Nine different antigens (plus soluble Leishmania antigen as a positive control) were tested as vaccine candidates in murine models for Leishmania. Adjuvants used were monophosphoryl lipid (MPL, donated by Corixa corporation) and interleukin 12 (IL-12, donated by Genetics Institute). The results of these studies were, however, inconclusive largely due to technical problems regarding the stability and potency of the antigens. The need for guidelines concerning the characterization and testing of recombinant antigens were discussed (see conclusions).

Corresponding address: Eric Dumonteil, Ph.D. Laboratorio de Parasitología, Centro de Investigaciones Regionales, Universidad Autónoma de Yucatán, Ave. Itzaes \# 490 x 59, C.P. 97000, Mérida, Yucatán, Mexico. Tel/Fax: (52-9) 924-59-10 Email: oliver@tunku.uady.mx 


\section{E Dumonteil, D McMahon-Pratt, VL Price.}

The individual presentations on second generation Leishmania vaccine candidates suggested several promising candidates that have shown protection in animal studies. Some of these presentations are summarized below.

\section{Antigens that show protection in animal models}

Nicolas Fasel of the University of Lausanne, Lausanne, Switzerland and Sima Rafati of the Institute Pasteur in Tehran, Iran, described vaccination studies against cutaneous Leishmania (L. major) done in mice and African green monkeys. The antigen used was L. major histone $\mathrm{H} 1$, a nuclear antigen that has no homology to human H1. Antigen was made either as a recombinant glutathione S-transferase (GST)fusion protein in E. coli or as a long synthetic peptide representing the complete histone $\mathrm{H} 1$ sequence. Montanide ISA 721 was used as adjuvant. Elisa data showed that both antigens elicited an increase in specific antibodies after vaccination, some (but not all) monkeys had increase gamma interferon (IFN-gamma) levels, and all exhibited a positive delayed type hypersensitivity (DTH) response.

A DNA vaccine was also prepared encapsulated in microspheres, small particles of poly-lactide-co-glycolide. DNA encoded antigens were histone $\mathrm{H} 1$, cysteine proteinase $\mathrm{B}(\mathrm{CPB})$, and cysteine proteinase A (CPA), alone or in combination. A single injection of $20 \mu \mathrm{g}$ DNA was used for vaccination of mice. Only the cocktail containing CPA and CPB showed protection.

J. Alexander (University of Strathclyde, Glasgow, Scotland) studied the vaccine potential of enzymatically active and inactive CPB to protect against $L$. mexicana, and showed that vaccination with CPB alone may stimulate interleukin 4 (IL4) production and exacerbate infection. However, the addition of IL-12 to these active and inactive $\mathrm{CPB}$ recombinant antigens resulted in significant inhibition of lesion growth in CBA/Ca, BALB / c and $\mathrm{C} 57 \mathrm{BL} / 6$ mice following challenge infection. A DNA vaccine consisting of genes for the antigens
CPB, GP63 and GP46 has been tested in a murine model against $L$. mexicana by $E$. Dumonteil of the Universidad Autónoma de Yucatán, Merida, Mexico. The individual antigens showed limited protection, but a high dose $(50 \mu \mathrm{g}$ each) of the combination of all three antigens showed strong protection in initial studies.

G. Grimaldi and R. Porrozzi (FIOCRUZ, Rio de Janeiro, Brazil) and A. Campos-Neto (Infectious Disease Research Institute, Seattle, WA, USA) have examined rhesus macaques (Macaca mulatta) as an experimental model for study of cutaneous leishmaniases (CL) induced by either L. major, or L. amazonensis, or L. braziliensis infection. The rhesus macaques as a model for human disease is thought to be appropriate in that the outcome of Leishmania infection in primates is similar to that in humans in that it largely depends on the virulence of the infecting parasite strain and the immune responsiveness of the host. Lesion development, histopathology of CL and the Th1-type profile are similar to the human disease.

Several antigen/adjuvant combinations were tested for the induction of protection in both mice and monkeys to Leishmania challenge. The $L$. major antigens tested were: LeIF (ribosomal initiation factor), STI1 (a stress and temperature inducible protein), and TSA (a thiol-specific antioxidant).

Mice were immunized with a di-fusion comprised of TSA-LmSTI1 and a tri-fusion comprised of TSA-LmSTI1-LeIF. Protection was seen with either the di-fusion or the tri-fusion with IL12 as adjuvant but not with several other adjuvants tested.

Monkey immunizations were performed with TSA and LmSTI11 as antigens and IL-12 plus alum as the adjuvant. (Alum with IL-12 does not appear to change the Th1 pattern normally seen with IL12 as adjuvant but seems to make the antigen more immunogenic.) Three immunizations of $25 \mu \mathrm{g}$ each antigen were given with a challenge six weeks after the last immunization. Protective immunity followed immunization, in that no lesions 
4th TDR/IDRI meeting on 2nd. generation vaccine against Leshmaniasis.

developed in any of vaccinated monkeys $(n=6)$ after infection. (All controls developed lesions.) Monkeys vaccinated with LmSTI1-TSA+IL$12+$ alum were also protected against rechallenge with L. major (4 months following the first challenge), but no cross-protection was found against tertiary challenge 2 months following the second challenge with $L$. braziliensis. The data point to a long-lasting anti-L. major immunity induced in primates by the recombinant antigens LmSTI1 and TSA.

G. Matlashewski of McGill University (Montreal, Canada) described the A2 genes of Leishmania, which encode proteins of unknown function. The A2 genes are part of a multigene family of at least 11 genes and the protein is composed largely of multiple repeats of a 10 amino acid sequence. The protein is abundant in amastigotes and absent in promastigotes. It is present in L. infantum, L. chagasi, L. mexicana, $L$. amazoniensis, and $L$. donovani but not in $L$. tropicana and L. major. (L. major has been shown to have the A2 genes but in truncated form.)

The role of the A2 proteins has been studied both by creating knock-out strains ( 8 of the 11 genes were knocked out) and by using anti-sense technology. It was then shown that the A2-deficient strains of $L$. donovani were avirulent in mice. Thus, A2 can be considered an amastigote specific virulence factor and therefore a good vaccine candidate for $L$. donovani.

A2 was tested as a vaccine (recombinant protein or DNA vaccine) in a murine model of visceral leishmaniasis. Mice vaccinated with A2 protein showed greatly reduced parasite numbers in the spleen and a mixed Th1/Th2 type of response (increased IFN-gamma by spleen cells and no increase of IL4 but increased IgG2a and IgG1 levels). A2 as a DNA vaccine gave partial protection (measured by reduced LDU).

C.B. Palatnik de Sousa (University of Rio de Janeiro, Rio de Janeiro, Brazil) studied the fucose mannose ligand (FML) antigen complex of $L$. donovani and its GP36 component as a vaccine.
After demonstrating the protective capacity of FML and GP36 with saponins as adjuvant in mice, a dog vaccination trial was initiated (4). The FMLvaccine was examined in dogs naturally exposed in an endemic area in Natal, Brazil. Ninety-seven percent of the vaccinated dogs produced antibodies and $100 \%$ demonstrated a DTH response to the FML antigen seven months post-vaccination. After 2 years of monitoring the dogs, data indicate that significant protection was achieved: only $8 \%$ of vaccinated dogs showed mild signs of visceral leishmaniasis, with no deaths, while in the control group 33\% of the dogs developed clinical symptoms or fatal disease. After 41 months, 3/4 of the control dogs were infected (as determined by PCR and bone marrow puncture) and the remaining vaccinated dogs were all negative for parasites and continued to maintain positive antibody and DTH responses. Thus, the FMLvaccine induced significant protection against canine visceral leishmaniasis in the field.

D. Smith (Imperial College, London, UK) described the hydrophilic acylated surface antigens (HASPs) as vaccine candidates. HASPs are dually acetylated at their N-termini, a modification required for subcellular targeting within the parasite to the membrane. Although the precise function(s) of these proteins are not known, genetic experiments using knock-out and over-expression mutants clearly indicate a role in virulence of $L$. major. Although all of the proteins family members are expressed in metacyclic promastigotes, only HASPA1 and B are detectable in amastigotes.

HASPs, expressed as unmodified recombinant proteins in E.coli, were employed for immunization of BALB/c mice (with or without IL-12). Immunization resulted in protection against infection with $L$. donovani in the animal model of visceral leishmaniasis (5). HASPB 1 induces a low level of IL-12 production in murine dendritic cells. Protection correlates with the level of IFN-gamma producing CD8 $\mathrm{T}$ cells and $\mathrm{IgG} 1$ production. Interestingly, rHASPB 1 fails to confer protection in IL-4 and IL-4 receptor alpha (IL-4R) deficient 


\section{E Dumonteil, D McMahon-Pratt, VL Price.}

mice. These data suggest further complexity in the regulation of vaccine-induced immunity.

\section{Understanding mechanisms of protection}

P. Scott of the University of Pennsylvania (Philadelphia, PA, USA) discussed studies on the mechanism of protection from leishmaniasis which were done in a Vervet monkey model (6). The antigen used was ALM (autoclaved L. major) with IL-12 as adjuvant. The immunizations resulted in increased specific IFN-gamma levels and a typical Th1-type response. Specific IFN-gamma levels were as high as the positive controls, which were animals that had already healed and were resistant. However, the vaccine did not protect. The conclusion from this study was that IFN-gamma production by PBLs to crude leishmanial antigen does not always correlate with immunity.

He then discussed the role of IL-12 as one of several parameters that might be required for eliciting and maintaining immunity (7). It was shown that IL-12 knockout mice, if treated with IL-12 exogenously, are resistant to L. major infection. However, upon rechallenge after cessation of IL12 treatment, they became susceptible. In addition, adoptive transfer of Th1 cells from healed mice into IL-12 knockout mice failed to protect against challenge. Moreover, the IFN-gamma response is not recovered in the IL-12 knockout mice. Thus, IL-12 seems to be needed continuously to maintain resistance. This might be due to a requirement for IL-12 to optimize IFN-gamma production from Th1 cells, to protect Th1 cells from apoptosis, to promote Th1 cells survival, and/or to recruit additional Th1 cells from a naïve or undifferentiated $\mathrm{T}$ cell population.

D. McMahon-Pratt (Yale University, New Haven, CT, USA) discussed severity of disease as a function of the infecting Leishmania species together with host genetics and consequent inflammatory and immune responses. In the case of cutaneous murine leishmaniasis, infection caused by L. amazonensis appears to be readily distinguished from that induced by L. major in a number of significant immunologic features. For L. amazonensis, the mechanisms involved in pathogenesis in the murine model appear to singularly involve immune subversion and require the participation of both CD4+ T cells and B cells (immunoglobulin). A low level of IFN-gamma (with little/no IL-4) production seems to be a feature of disease. Antigen presentation and monocyte recruitment at the local site of infection appears to be critical for the development of pathology. The roles of immune $\mathrm{T}$ and $\mathrm{B}$ cells in pathology as well as resolution of disease could impact the efficacy of vaccines against New World leishmaniasis, especially potentially as the immune response wanes after vaccination.

A. Campos-Neto (Infectious Disease Research Institute, Seattle, WA, USA), pointed out that for the past 20 years, it has been generally accepted that immunity against experimental infection of mice with Leishmania is strictly dependent of the generation of a Th1 type of immune response against the parasite's antigens. However, they have observed that several leishmanial recombinant antigens, selected on this criteria and expressed as recombinant proteins, do not necessarily turn out to be protective antigens. For example, BALB/c mice infected with $L$. major develop low antibody titers to the antigen Ldp23, and to many others tested. In addition, Ldp23 induces preferentially Th1 response in lymph node cells from infected mice. However, in combination with adjuvants that preferentially induce Th1 responses such as IL-12 and MPL-SE, despite stimulating strong antigen specific Th1 response in the absence of any detectable Th2 response, Ldp23 does not confer protection. In contrast, the antigen LACK stimulates strong and preferentially Th2 responses in lymph node and spleen cells from $L$. major-infected mice. In addition the sera of these animals contain high titers of IgG1 anti-LACK antibodies. In spite of this, LACK induces substantial protection in BALB/c mice if administered in conjunction with adjuvants that stimulate Th1 responses. In another situation, antigens like LmSTI1 stimulate strong and

\section{Revista Biomédica}




\section{4th TDR/IDRI meeting on 2nd. generation vaccine against Leshmaniasis.}

preferentially Th1 responses in lymph node cells of $\mathrm{BALB} / \mathrm{c}$ mice infected with $L$. major and the sera of these animals contain high titers of both IgG1 and IgG2a anti-LmSTI1 antibodies. Moreover, LmSTI1 induces excellent protection in these animals if used with IL-12 or MPL-SE as adjuvants.

Therefore, for antigen selection purposes in vaccine development against leishmaniasis these results do not support the Th1 paradigm, although a Th1 response may well be essential for protection against leishmaniasis. In conclusion, for vaccine development against leishmaniasis, the polarization to Th1/Th2 antigen-specific immune response that is developed against the parasite antigens during the infectious process may be irrelevant. Rather, immunogenicity and perhaps the amount of antigen expressed or secreted by the parasite in vivo, are more important factors reflecting protective anti parasite immune response. Antigens that fulfill these criteria should therefore be more successful in inducing protection as long as they are administered with adjuvants that in combination with them modulated a strong Th1 response.

M. Barral-Neto (Universidade Federal de Bahia, Bahia, Brazil) described a priming in-vitro (PIV) system that was used to evaluate the immune response of naïve individuals following in-vivo vaccination with parasite. IFN-gamma levels were followed as an indicator of the type of response to Leishmania antigen. Peripheral blood mononuclear cells (PBMC) of volunteers who were DTHnegative and anti-Leishmania antibody-negative were used for in-vitro stimulation with Leishmania antigen and IFN-gamma was monitored. This invitro stimulation experiment identified two different types of responders: those that produced low amounts of IFN-gamma and those that produced high levels of IFN-gamma. The IFN-gamma levels were directly proportional to tumor necrosis factoralpha (TNF-alpha) and interleukin-10 (IL-10) levels but not to interleukin-5 (IL-5) levels. Those volunteers who produced low amounts of IFNgamma in vitro remained low producers 40 days after vaccination, whereas high producers exhibited increased IFN-gamma production after vaccination. However, at 6 months post-vaccination, all individuals tested were producing similarly high levels of IFN-gamma upon stimulation of their PBMC with Leishmania promastigotes, indicating that the low in-vitro producers were slow in-vivo responders to vaccination. High IFN-gamma producers exhibited an increased frequency of activated CD8+T cells both in-vitro and in-vivo, as compared to low IFN-gamma producers. This delay in the cell mediated immune (CMI) response may influence resistance to leishmaniases.

\section{New antigen discovery}

T. Aebischer, from the Max Plank Institute in Tubingen, Germany, discussed the generic characteristics of potential new protective antigens. Previous work (4) indicated that protective antigens require a minimal level of expression of $10^{5-6}$ molecules per organism throughout the parasite lifecycle. In addition, protection seemed to be dependent upon antigen accessibility, i.e. a molecule expressed on the surface membrane or secreted.

Studies of the kinetics of antigen presentation in infected mice, examined using green fluorescent protein (GFP)-labeled parasites, confirm these observations. They have found two waves of antigen presenting cells in high dose infection in the draining lymph node with only the second harboring live parasites. Therefore, in natural low dose infection protective antigens presented from live parasites should be accessible throughout infection and thus would most likely be secreted or surface components. These results strengthen the conclusion that in the rational search for candidate vaccine antigens, they should be abundant, preferably secreted or surface exposed protein molecules.

J. Blackwell (Wellcome Trust Centre for Molecular Mechanisms in Disease, Cambridge, UK) described a microarray analysis of the Leishmania life cycle as a mechanism for identifying new potential vaccine antigens. In the vertebrate host, metacyclic promastigotes rapidly enter macrophages and transform to amastigote forms. They used DNA 


\section{E Dumonteil, D McMahon-Pratt, VL Price.}

microarrays for simultaneous monitoring of expression profiles for 2183 unique Leishmania genes as the parasite undergoes the developmental transition from logarithmic promastigotes to metacyclic forms and host-derived amastigotes. From this analysis they have identified over 100 previously unknown genes which are upregulated in amastigotes. These are presently being tested as new vaccine candidates. Some cocktails of these antigens appear to be effective as DNA vaccines in mice.

\section{CONCLUSIONS.}

Based on these presentations, it was concluded that several promising Leishmania vaccine candidates exist that warrant further development, eventually towards human clinical testing. However, it was recommended that strict quality control of recombinant antigens should be included in pivotal animal studies (as well as for monkey and human trials). Antigen characterization should include: SDS-PAGE (reduced and non-reduced), Western blot analysis, endotoxin levels, sterility and amino acid analysis for determination of protein concentration. It was agreed that the minimum criteria for moving a candidate vaccine to phase I studies must include the consistent (i.e. repeated in a second independent laboratory) induction of significant protection in an animal model, and a formulation with an adjuvant than can currently be used in clinical trials. It was felt that collaboration and coordination of efforts in the testing of vaccine candidate antigens in more than one laboratory is warranted.

Additionally, though there have been important development in this field, we still do not understand completely what constitutes a protective response in humans. It is thus considered important to encourage continued effort towards understanding what constitutes long-term protection to leishmaniases both in animal models as well as in humans. Defining the correlates of protection and development of predictive assays for the evaluation of vaccine candidates are important to the progress of the field.

\section{REFERENCES.}

1.- Sharifi I, FeKri A R, Aflatonian M R, Khamesipour A, Nadim A, Ahmadi-Mousavi M R, et al Randomised vaccine trial of single dose of killed Leishmania major plus BCG against anthroponotic cutaneous leishmaniases in Bam, Iran. Lancet 1998; 351: 1540-3.

2. Modabber F. First generation leishmaniases vaccines in clinical development: Moving, but what next? Curr Opin Anti-infect Invest Drugs 2000; 2: 35-9.

3.- Velez I D, del Pilar Agudelo S, Arbelaez M P, Gilchrist $\mathrm{K}$, Robledo S M, Puerta J A, et al. Safety and immunogenicity of a killed Leishmania (L.) amazonensis vaccine against cutaneous leishmaniasis in Colombia: a randomized controlled trial. Trans R Soc Trop Med Hyg 2000; 94: 698-703.

4.- da Silva VO, Borja-Cabrera G P, Correia Pontes N N, de Souza E P, Luz K G, Palatnik M, et al. A phase III trial of efficacy of the FML-vaccine against canine kala-azar in an endemic area of Brazil (Sao Goncalo do Amaranto, RN). Vaccine 2000; 19: 1082-92.

5.- Stager S, Smith D F, Kaye PM. Immunization with a recombinant stage-regulated surface protein from Leishmania donovani induces protection against visceral leishmaniasis. J Immunol 2000; 165: 7064-71.

6.- Gicheru M M, Olobo J O, Anjili C O, Orago A S, Modabber F, Scott P. Vervet monkeys vaccinated with killed Leishmania major parasites and IL-12 develop a type 1 response, but are not protected against challenge infection. Infect Immun 2001; 69: 245-51.

7.- Park A Y, Hondowicz B D, Scott P. IL-12 is required to maintain a Th1 response during Leishmania major infection. J Immunol 2000; 165: 896-902.

8.- Aebischer T, Wolfram M, Patzer S I, Ilg T, Wiese M, Overath P. Subunit vaccination of mice against new world cutaneous leishmaniasis: comparison of three proteins expressed in amastigotes and six adjuvants. Infect Immun 2000; 68:1328-36.

\section{Revista Biomédica}

